\title{
Dynamic Routing Method over Hybrid SDN for Flying Ad Hoc Networks
}

\author{
Alaa Taima Albu-Salih ${ }^{1} \quad$ Seyed Amin Hosseini Seno ${ }^{{ }^{*}}$ \\ Saad Jasim Mohammed ${ }^{2}$
}

Received 3/5/2018, Accepted 2/9/2018, Published 13/9/2018

This work is licensed under a Creative Commons Attribution 4.0 International License.

\begin{abstract}
:
Due to the high mobility and dynamic topology of the FANET network, maintaining communication links between UAVs is a challenging task. The topology of these networks is more dynamic than traditional mobile networks, which raises challenges for the routing protocol. The existing routing protocols for these networks partly fail to detect network topology changes. Few methods have recently been proposed to overcome this problem due to the rapid changes of network topology. We try to solve this problem by designing a new dynamic routing method for a group of UAVs using Hybrid SDN technology (SDN and a distributed routing protocol) with a highly dynamic topology. Comparison of the proposed method performance and two other algorithms is simulated. The simulation results show that the proposed method has better results than traditional algorithms in the package delivery ratio, average end to end delay, packet loss, throughput and normalized routing Load.
\end{abstract}

Keywords: FANET, Hybrid SDN, IoT, OLSR, Routing.

\section{Introduction:}

Advances in electronics and computers, communication sensors and communications have led to the development of remotely driven drones that can fly independently or can be controlled remotely without transporting any human power. Since these UAVs have flexibility, easy installation, and relatively small operating costs, the use of unmanned aerial vehicles (UAVs) provides new ways for both military and non-military applications, such as search and destruction operations, border surveillance, fire management, disaster monitoring, remote sensing and traffic monitoring (1). Flying adhoc networks (FANETs) can be considered a form of MANET network, where nodes are defined as UAVs. According to this definition, the UAV system can be a valid FANET not only for multi-drone systems, but also the UAV's connection should be completed with the help of an inter-drone ad hoc network.

Therefore, if the relationship between UAVs is fully based on the drone infrastructure link, it can be considered as a FANET (2).

By this definition, FANET is a form of MANET, and there are many common design considerations between the two. Additionally, MANET can also be classified as a subset of VANET, which is also a subgroup of MANET. This relationship is shown in Fig.1(3).

\footnotetext{
${ }^{\mathrm{T}}$ Ferdowsi University of Mashhad, Iran.

${ }^{2}$ Ministry of Education, Iraq.

*Corresponding author: hosseini@um.ac.ir
}

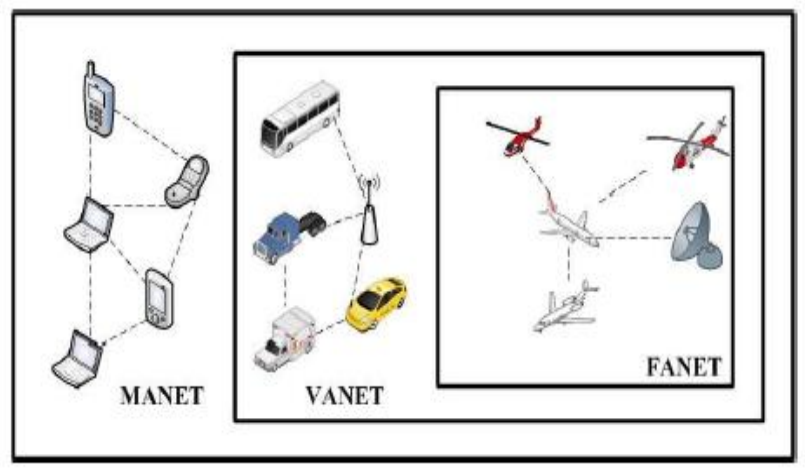

Figure 1. MANET, VANET, and FANET Networks

The most significant difference between the FANETs and, other adhoc networks, is the mobility of the nodes. The MANET nodes movement is relatively slow compared to VANET. In FANET, the degree of mobility of the node is much higher than VANET and MANET. A UAV has a speed of $30-460 \mathrm{~m} / \mathrm{s}$, and this situation has other challenges (4).

While the MANET node moves on the ground, the VANET node moves on the highway, and the FANET nodes float in the sky. MANETs generally implement a random waypoint model (5), in which the direction and speed of the nodes are randomly selected. The VANET node is limited to moving on the highway or the road. Therefore, VANET's mobility models are highly predictable. Table 1 shows the differences between MANET, VANET, and FANET(6). 
Table 1. Differences between MANET, VANET and FANET

\begin{tabular}{|c|c|c|c|}
\hline Parameter & MANET & VANET & FANET \\
\hline Node mobility & Low & High & Very high \\
\hline Mobility model & Random & Regular & $\begin{array}{l}\text { Regular for predetermined paths, but special } \\
\text { mobility models for autonomous multi-UAV } \\
\text { systems }\end{array}$ \\
\hline Node density & Low & High & Very low \\
\hline Topology change & Slow & Fast & Fast \\
\hline $\begin{array}{l}\text { Radio propagation } \\
\text { model }\end{array}$ & Close to ground & Close to ground & High above the ground \\
\hline LoS & $\begin{array}{l}\text { LoS is not } \\
\text { available for all } \\
\text { cases }\end{array}$ & $\begin{array}{l}\text { LoS is not available for } \\
\text { all cases }\end{array}$ & LoS is available for all cases \\
\hline $\begin{array}{l}\text { Power consumption } \\
\text { and network lifetime }\end{array}$ & $\begin{array}{l}\text { Energy efficient } \\
\text { protocols }\end{array}$ & Not needed & $\begin{array}{l}\text { Energy efficiency for mini UAVs, but not } \\
\text { needed for small UAVs }\end{array}$ \\
\hline Localization & GPS & GPS,AGPS, DGPS & GPS, AGPS, DGPS, IMU \\
\hline
\end{tabular}

The optimal route and deployment of UAVs (drones) are used as the main air stations to collect data from the Internet of Things (IoT) (7). Indeed, drones can play a key role on the IoTs, which consist of devices with a limited battery size, such as sensors and health monitors (8). Because of their energy constraints, these devices can not normally be transmitted over long distances. In such IoT scenarios, drones can move dynamically towards IoT devices, collect IoT data and transfer it to other devices that are outside the communication ranges of the IoT devices, as seen in Fig. 2. In this case, UAVs play the role of mobile collectors for IoT networks. However, for the effective use of UAVs for IoT communications, there are a number of challenges to consider, such as optimum deployment, optimal route, data routing, user participation, air-to-ground modeling, and the efficient use of energy for UAVs and IoTs.

Software-defined networks (SDN) can be used in the IoT and the UAV networks. The separation of the control plane and data forwarding plane is the foundation of the SDN, which can bring benefits to these networks. First, the centralized controller maintains an overall view of the network, which reduces power consumption by sensors. Second, it improves the efficiency of the routing algorithm according to the exact location information (9).

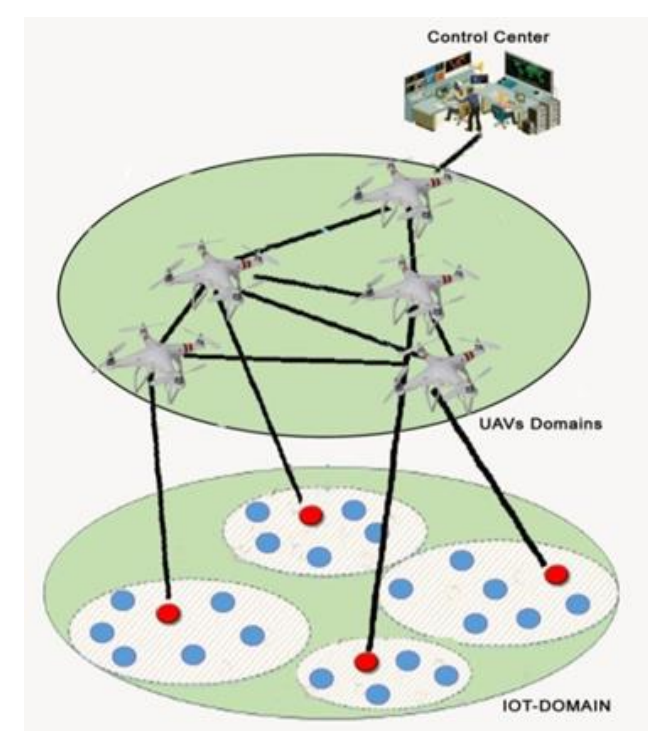

Figure 2. The Communication between UAVs and IoT devices.

The FANET network is characterized for high node mobility, rapid network topology change, intermittent links, energy constraints, and link quality changes. Traditional routing algorithms are not able to meet FANET requirements and perform missions collectively in this situation; quality of service-based routing algorithms can only be used under certain conditions. So we present a new dynamic routing strategy based on the Hybrid SDN (part of the nodes on the SDN network can dynamically migrate from SDN to a distributed traditional protocol (eg OLSR) and deny SDN forwarding rules).

The rest of the article is as follows: In the second section, a review of routing methods in FANET networks will be presented. In Section 3, the steps of the proposed strategy are presented. Section 4 describes the limitations and assumptions of the networks. In the fifth section, the simulation and evaluation of the proposed framework are discussed and in the final section we will look at the results and future work. 


\section{Related works:}

Routing is the only problem that remains active on all types of networks. For FANET networks, robust and error-tolerant routing protocols are required that can provide a minimum delay during route selection, efficient path reconnection, fast recovery, improved control over delays and jitter, and provide higher quality service to end-users (10).

There are some articles that look at the use of routing protocols for potential use in FANET networks. Although typical ad hoc routing protocols are designed for mobile nodes, they are not necessarily suited to FANET nodes due to different dynamic and interruptible needs. Therefore, there is still a need for a routing protocol according to the specific needs of the FANET, which is consistent with high upgrades, dynamic topologies, and different routing capabilities. The routing protocols are trying to increase the packet delivery ratio, reduce the delay, and consume resources. In addition, we must consider problems of scalability, loop freedom, energy conservation, and efficient use of resources (11).

Kuldeep et al. in (12), analyzed the AODV, DSDV, and OLSR routing protocols under different parameters, including end2end delay, throughput, and packet delivery ratio for FANET network. Simulation results can be seen that the OLSR routing protocol is better than the other two AODV and DSDV routing protocols in terms of end-to-end delay, throughput and packet delivery ratio.

Leonov in (13) describes the solution to the problem of routing for FANET, and provides a comparative analysis of the existing FANET algorithms and two algorithms based on swarm intelligence (colonies of ant and honey bees). The comparison results show that solutions based on AntHocNet and BeeAdHoc-inspired biology algorithms are better than traditional AODV, DSDV, and DSR routing algorithms.

Yu. et al. (14), proposed an ant optimization-based, polymorphism-based routing algorithm for the APAR algorithm to solve routing problem in FANET. This algorithm combines the ACO algorithm and the dynamic source routing algorithm (DSR). The levels of pheromone in the paths found in the routing discovery process is selected as the standard for selecting the route, and by calculating the route distance, one can determine the loading level of a route, and track stability. A new pheromone volatilization algorithm is also introduced into the algorithm. Meanwhile, the algorithm can change the formation of the air force to prevent compromised network performance. The simulation results show that the APAR algorithm is based on traditional algorithms in data packet delivery ratios, end2end delay, routing overhead, and reliability in the battlefield environment.

Rosati et al. (15) compared the performance of POLSR and OLSR in FANET, consisting of fixed drones. Such networks are characterized by a high degree of mobility that challenges the routing protocol. Routing protocols designed for MANETs have often failed in tracing the evolution of network topologies. They solve this problem by creating an OLSR extension called P-OLSR: the use of GPS information to predict the quality of wireless links evolves. Simulation of networks and field experiments confirm the expectations. With POLSR, routing is looking for uninterrupted topology changes, which is not the case with OLSR.

\section{Material and Methods: \\ The Proposed Method:}

To address the challenge of routing in FANET network, we used a hybrid-SDN controler, in which network control is split between SDN control and UAVs (data plane). In this sense, we allowed (UAVs) the decisions to transport data in a distributed manner, without the intervention of the SDN controller. Traditional (non-SDN) routing protocols such as OLSRs can be used to provide robust network architecture to discover network topology and routing in the presence of network defects and mobility.

In this way, some of the nodes in the SDN network can dynamically migrate from SDN to a traditional distributed protocol (eg OLSR) and rule out SDN forwarding rules. For example, nodes can be selected in a specific area where frequent network changes occur. Naturally, the distributed protocol will be compatible with these changes faster than the remote control SDN. When conditions change, for example, the connection to the controller becomes more stable, nodes can be re-configured by the SDN controller and migrate back to the SDN.

By transferring the logical level of control to the UAV nodes, we make the SDN architecture more suitable for the network environment (UAV) and make it more compatible with network modifications. In addition, the proposed network architecture offers a potential application for advanced routing policies for a highly dynamic topology UAV.

Based on the limitations and assumptions of UAVs in the IoT network, suggested methods for designing a new dynamic routing algorithm for a group of UAVs using the Hybrid SDN can be presented as follows:

- Providing a new framework to optimize the multiUAV routing strategy for the IoT network with 
respect to the dynamic topology of FANET network and is exploited by the Hybrid SDN framework.

- This framework consists of three main modules: topology discovery module, statistics gathering module and route computation module. As shown in Fig. 3, all of these three modules are located in the SDN controller. Below we will explain each of them in detail.

- Topology Discovery Module: This module collects periodic information about network topology, including nodes and links. Network connectivity information is constantly available to other service modules in the controller.

- Statistics Gathering Module: Monitors the network in predefined slots. This module collects the status of the wireless network connection status (for example, RSSI, delay, bit error rate) and flight statistics (such as speed, GPS data, IMU data). The information for this module is sent as a reference to create a new flow path.

- Route Computation Module: The module calculates the optimal path. This module uses the information obtained from previous modules to solve the problem and find the optimal path based on link costs.

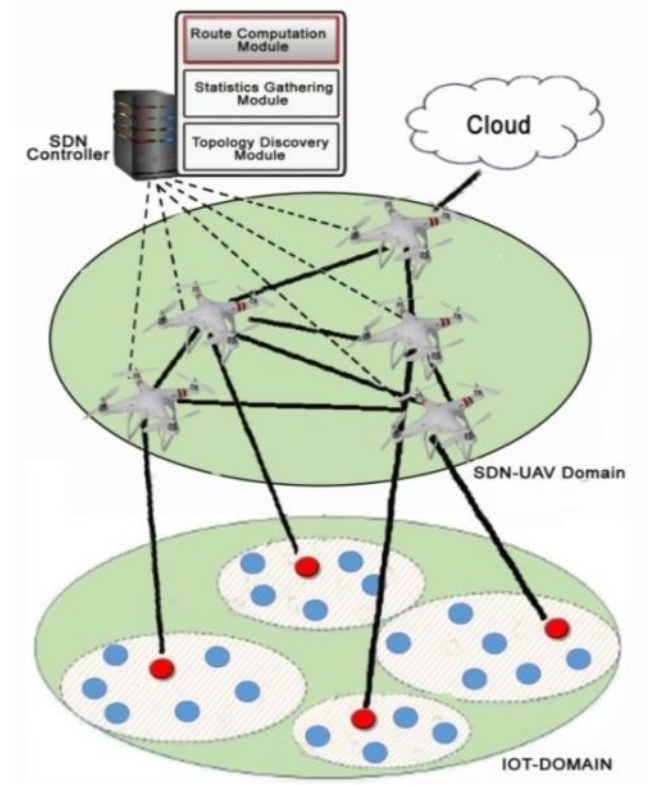

Figure3. SDN-based UAV-IoT Network Topology

- Each UAV is equipped with both LTE and WiFi interfaces. The control link between SDN and UAV controls is enabled through LTE connectivity, while data communication between the UAVs and IoT devices is provided via the WiFi link.

- We used the OLSR routing protocol for data traffic for the lack of access to the SDN controller. The use of OLSR provides an appropriate response to change the topology events, such as adding/removing UAV nodes and wireless links among them.

\section{Assumptions and Limitations:}

Assumptions and limitations in this study we will consider are as follows:

- Assumptions and Limitations of the IoT Network:

oLayout of IoT nodes in the network: Because many of IoT network applications, nodes on the network have been distributed uniformly, we also assume that the distribution of IoT nodes in the network will be uniform.

oThe IoT nodes are hierarchical: Nodes are of two types: $\mathrm{CH}$ (Cluster Head) and CM (Cluster Member). The $\mathrm{CHs}$ and $\mathrm{CMs}$ are randomly deployed in the network field.

oGround-to-air communication: Each device will typically have a LoS view towards a specific UAV with a given probability. This LoS probability depends on the environment, location of the device and the UAV, and the elevation angle between the device and the UAV.

oTransmission rates of IoT nodes: In this article, each of the IoT nodes is supposed to be the ability to set their own rates as well as the radius of the transmission.

oNodes place: all nodes of wireless sensor are aware of their geographical place based on Global Positioning System (GPS), and their locations are known to UAV and are used to find optimal route for UAVs.

- Assumptions and Limitations of the UAV Network:

oLayout of UAV nodes in the network: We assume that the distribution of UAV nodes in the network will be distributed in ad hoc formation.

oThe ability to move at fixed speed: In this article, every UAV has the ability to move at fixed speed.

oThe ability to move at fixed flying height: In this article, every UAV has the ability to move at fixed height.

The ability to do heavy calculations and processes: UAV with high processing power and memory will be assessed.

oThe presence of obstacles to the UAV: In this article, every UAV have the ability to move with no obstacles.

- The absence of UAVs collision: every UAV have the ability to move without risk of collision.

- Assumptions and Limitations of the SDN Network:

oLayout of SDN network: SDN network is formed by mounting the OpenFlow switches on the UAVs and the control facility on a centralized controller. 
OSDN aware of IoTs \& UAVs location: We considered a centralized network in which the locations of IoT devices and UAVs are known to a SDN controller. The controller is aware of the global network topology.

oCentral Controller: We used a single central controller which monitors the overall traffic network.

\section{Results and Discussion:}

In this section, we will compare the results of our work with the related work (14) and (15). These articles can be used to evaluate the effective evaluation of the proposed method for dynamic routing for FANET network. The parameters that are considered in this paper to evaluate the effectiveness of proposed method are divided into five main categories:

1. Package Delivery Ratio(PDF)

2. Average End to End Delay(AED)

3. Throughput

4. Packet loss

5. Normalized Routing Load(NRL)

\section{Simulation Settings:}

In this simulation, the size of the $600 \times 600$ square meter network is assumed. The evaluation was performed using Python software as a platform for implementation on a system with a core processor unit Core i5-2410M $2.30 \mathrm{GHz}$ and 4 gigabytes of main memory. The other simulation parameters are summarized in Table 2.

To implement the research, we developed the previous proposed SDN-based approach with with
Python language such POX controller and we used OpenFlow protocol to collect network statistical information from our network infrastructure. A routing module which got the statistical information to make a decision for data plane.

Table 2. Simulation Parameters

\begin{tabular}{ll}
\hline Parameter & Value \\
\hline The Network simulator & NS-2.35 \\
MAC Type & 802.11 \\
Radio Wave Propagation & TwoRayGround \\
Antenna & Omni Antenna \\
Simulation Time & 200 seconds \\
Mobility Model & Random Walk \\
Area size & $600 \times 600 \mathrm{~m} 2$ \\
No. of IoT Nodes & 100 \\
No. of UAV & 2101520 \\
Speeds of UAV & $10203040 \mathrm{~m} / \mathrm{s}$ \\
Heights of UAV & $70 \mathrm{~m}$ \\
Transmission Range & $40 \mathrm{~m}$ \\
UAV Elevetion Angles & $45 \mathrm{deg}$ \\
Transmission bit rate $\mathrm{f}$ & $200 \mathrm{kbps}$ \\
Packet size & $2000 \mathrm{bit}$ \\
\hline
\end{tabular}

2. Evaluation of the proposed framework in comparison with related work:

In this section, we will compare the proposed Hybrid-SDN framework with two algorithms in related tasks in terms of packet delivery ratio, endto-end delay, packet loss, troughput, and normalized routing load, and we will evaluate the following two scenarios. We consider two algorithms associated with the method presented in (14) and (15).

Scenario 1: Various Number of UAV Nodes 


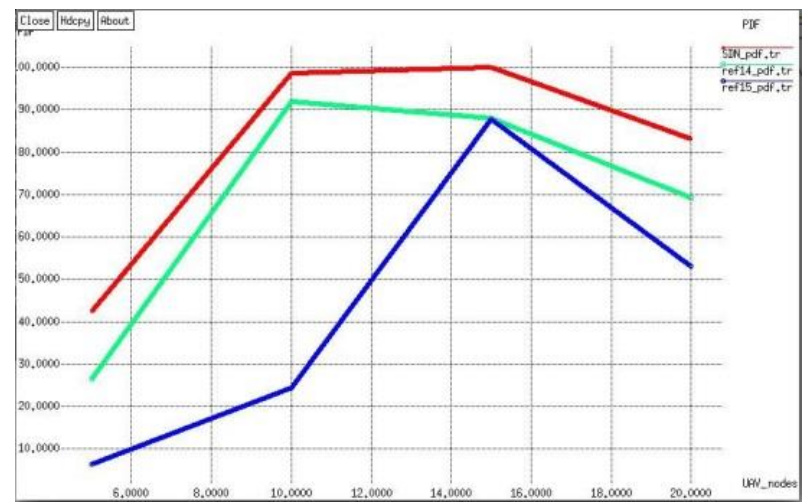

a) $\mathrm{PDF}$

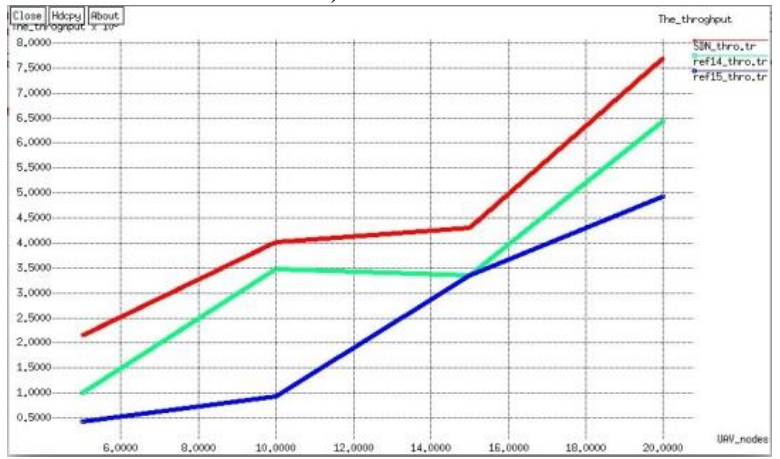

c) AED

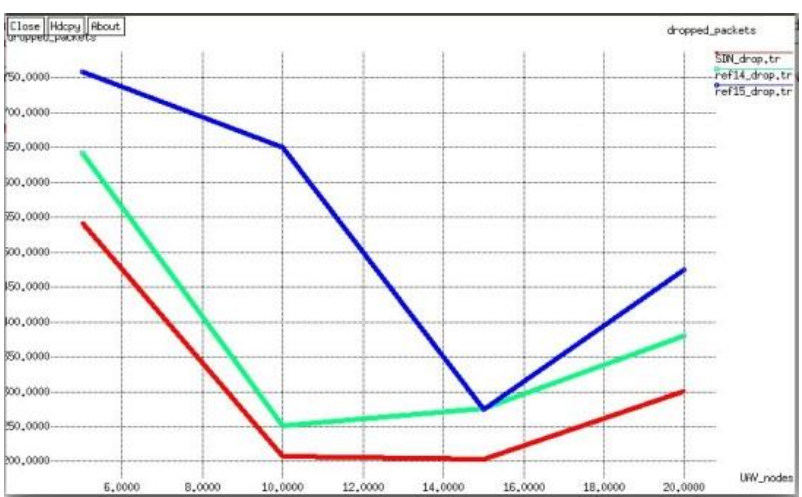

b) Drop Packets

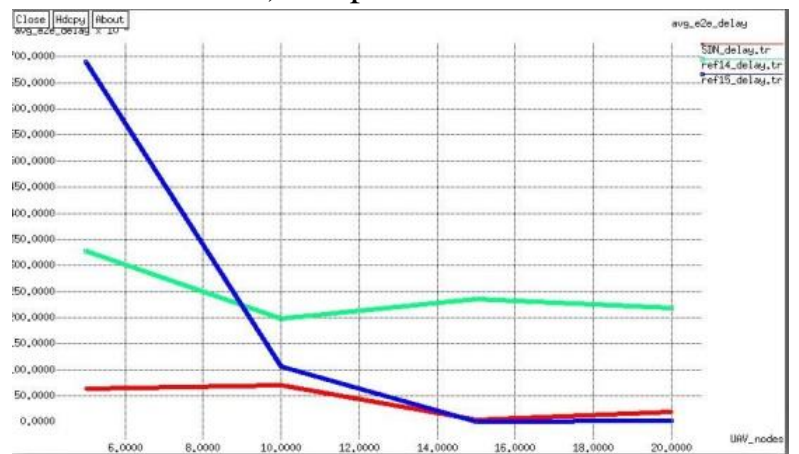

d) Throughput

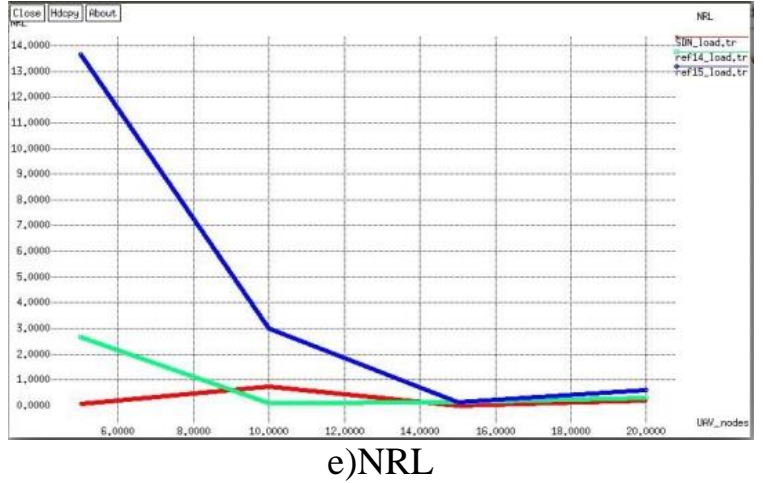

Figure 4. [a-e]. The performance metrics of Hybrid-SDN, Ref (14) and Ref (15) for scenario 1

Figure 4 [a-e] shows the performance metrics of proposed method-based hybrid SDN and two methed were propsed in (14) and (15) for scenario 1. Fig. (4a) shows the PDF of AODV is best in method based hybrid SDN. PDF in refereces (14) and (15) performed the worst. Fig. (4b) displays the number of drop packets in (14) and (15), which is higher than our proposed method. The packet losses are increased when the number of nodes decreased. Figure 4.c shows the AED is decreased when the number of nodes increased.
AED in our proposed method is least and in (14) and (15) is highest. In figure4.d, the throughput is more significant with our proposed method and the throughput is less significant with methods were proposed in (14) and (15). In figure 4.e, the NRL of these methods is decreased when the number of nodes increased. The NRL in method based hybrid SDN is low because the contoller decides the speed of the nodes and in (14) and (15) is high.

Scenario 2: Various Speed of UAV 


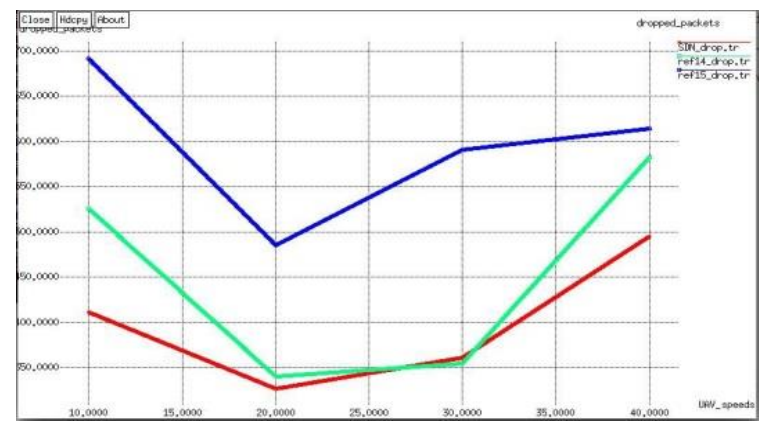

a) PDF

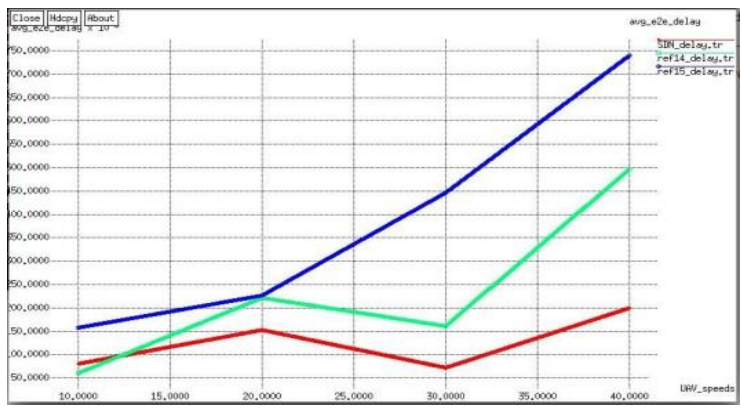

c)AED

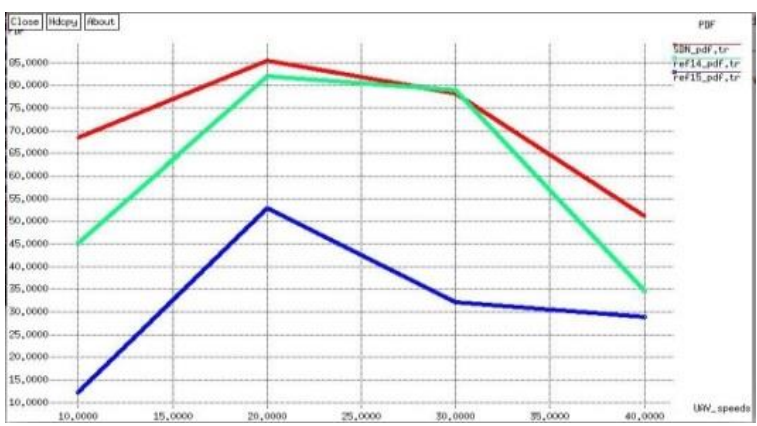

b)Drop Packets

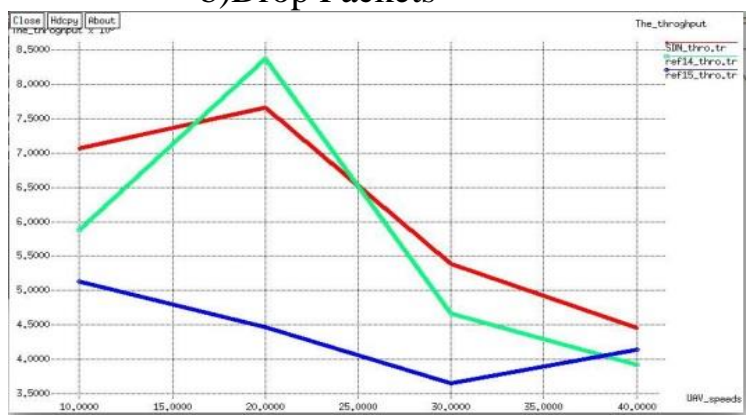

d)Throughput

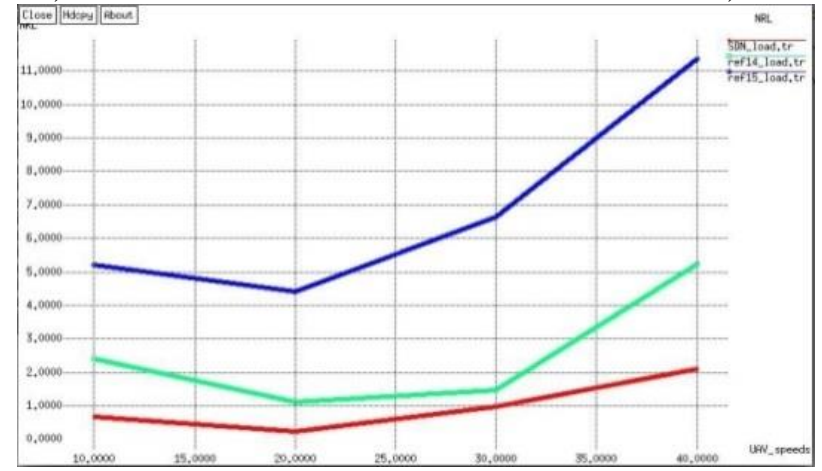

e) NRL

Figure 5. [a-e]. The performance metrics of Hybrid-SDN, Ref (14) and Ref (15) for scenario 2

Figure 5 [a-e] shows the performance metrics of proposed method-based hybrid SDN and two methed which were propsed in (14) and (15) for scenario 2. Figure 5a displays the PDF, which were decreased when the node speed was increased. The proposed method has high PDF while (14) and (15) are lower. Figure $5 \mathrm{~b}$ shows the number of packets lost in (14) and (15) is highest, while in our propsed method is lowest; the lost packet is increased when the node speed increased. Figure $5 \mathrm{c}$ shows the AED increases when the node speed increased. AED in proposed method is lowest and in (14) and (15) is highest. In figure $5 \mathrm{~d}$, the throughput of our proposed method were decreased when the node speed were increased. The proposed method has high throughput and PDF while the methods in (14) and (15) are lower. In Fig. (5e), the NRL of these protocols is increases with high speed for all methods. The method-based hybrid SDN has low NRL than other methods while the methods in (14) and (15) have high NRL.

\section{Conclusion:}

The FANET network is characterized by high node mobility, rapid network topology change, intermittent links, energy constraints, and link quality changes. Traditional routing algorithms are not able to meet multiple requests for UAVs and do missions collectively in this situation; QoS-based routing algorithms can only be used under certain conditions. So, we introduced a new dynamic routing Strategy based on Hybrid SDN (SDN controler and distributed routing protocol) to solve these problems. Simulation results showed that the proposed method can reduce the average delay, normalized routing load, and increase the throughput and packet delivery fraction.

\section{Conflicts of Interest: None.}

\section{References:}

1. Georgee J, Sujit P, Sousa J. Search strategies for multiple UAV search and destroymissions, J Intell Robot Syst, 2011; 61(14), 355-367. 
2. Moorse B, Enngh C, Goodrich M. UAV video coverage quality maps and prioritized indexing for wilderness search and rescue, 5th ACM/IEEE inter. conference on Human-robot interaction , IEEE Press, 2010; 227-234.

3. Wang C, Soohraby K, Li B, Danishmand M, Hu Y. A survey of transport protocols for wireless sensor net., IEEE Network, 2006; 20(3): 34-40.

4. Claapper J, Young J, Carrtwright J, Grimes J. Unmanned Systems Roadmap, Inter. Conference on Info Processing in Sensor Networks, IPSN, ACM, New York, NY, USA, 2012; 145-146,.

5. Alizadeh M, Grenberg A, Maltz D, Paadhye J, Patel P, Prabhakar B. Data center TCP, SIGCOMM Comput Commun Rev, 2010; 40(4): 63-7.

6. Bekmezci I, Sahingoz O, Temel S. Flying ad-hoc networks (FANETs): A survey, Elsevier, Ad Hoc Net., 2013; 11(3): 1255-1260,.

7. Dawy Z, Saad W, Ghosh A, Andrews J, Yaacoub E, Towards massive machine type cellular communications, IEEE Wireless Commn Magazine, 2017; 24(1): 120-128.

8. Hassanalieragh M, Page A, Soyata T, Sharma G, Aktas M, Mateos G, Kantarci B, Andreescu S. Health monitoring and management using internet-of-things (IoT) sensing with cloud-based processing: Opportunities and challenges, in Proc. Inter. Conference on Services Computingn, New York, USA, 2015; 285-292.
9. Tomovic S, Yoshigoe K, Maljevic I, Radusinovic I. Software-Defined Fog Network Architecture for IoT, Springer, Wireless Pers Commun, 2017; 92:181.

10. Sharma V, Kumar R. Cooperative frameworks and network models for flying ad hoc networks: a survey, John Wiley \& Sons, Ltd., 2016; 29(4): 102-138.

11. Gupta L, Jain R, Vaszkun G. Survey of Important Issues in UAV Communication Networks, IEEE Commn Surveys \& Tutorials , 2016; 18(2):11231152.

12. Kuldeep S, Anil K, Experimental analysis of AODV, DSDV and OLSR routing protocol for flying adhoc networks (FANETs), IEEE Inter Conference on Electrical, Comp and CommmnTech (ICECCT), 2015; 222-228.

13. Alexey V, Modeling of Bio-Inspired Algorithms AntHocNet and BeeAdHoc for Flying Ad Hoc Networks (FANETs), Inter Scien-Tech Conference on Actual Problems of Electronics Instrument Engineering (APEIE), 2016;1-10.

14. Yunlong Y, Le R, Wensheng C, Yaqing L, Qiangqiang Y, Kun F. Ant colony optimization based polymorphism-aware routing algorithm for ad hoc UAV network, Springer, Multimedia Tools and Applications, 2016; 75(22): 14451-14476.

15.Rosati S, Krużelecki K, Heitz G, Floreano D, Rimoldi B, Dynamic Routing for Flying Ad Hoc Networks, IEEE Transactions on Vehicular Technology, 2016; 65(3): 1690-1700.

\section{طريقة التوجيه الايناميكي لشبكات الطيران المخصصة باستخدام الثبكة المعرفة بالبرمجيات المهجنة
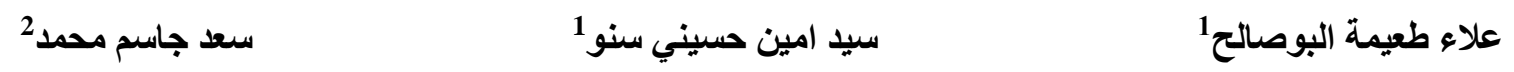

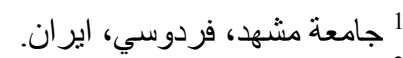

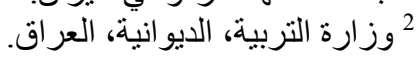

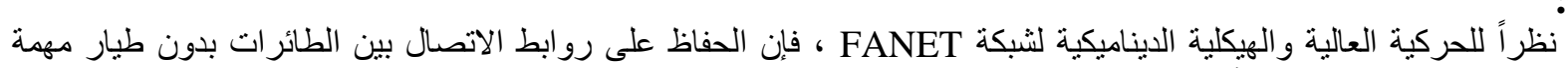

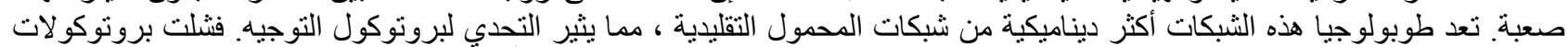

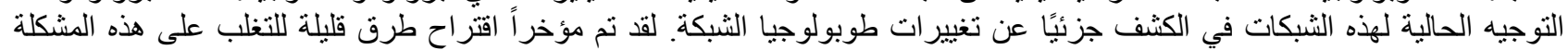

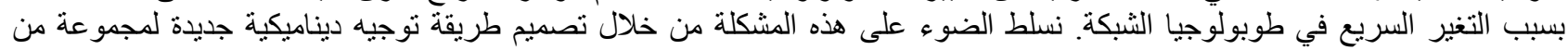

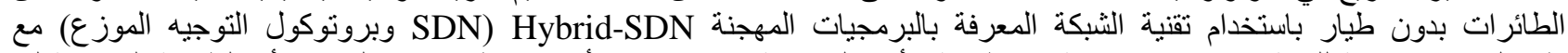

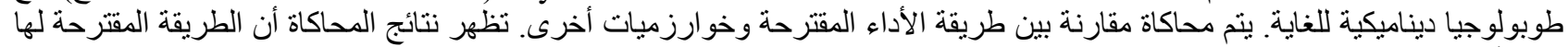

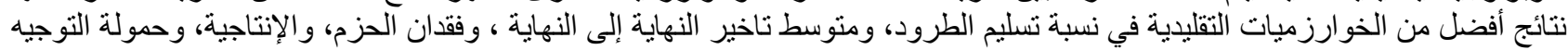
المعيارية.

الكلمات المفتاحية:شبكات الطير ان المخصصة ، إنترنت الأشياء، الثبكة المعرفة بالبرمجيات المهنة ،برنوكول OLSR، التوجيه. 